\title{
Presencia del Mapache Cangrejero Procyon cancrivorus (Carnivora: Procyonidae) en un agroecosistema del municipio de Santa Rosa de Cabal, Risaralda, Colombia
}

\author{
Juan Camilo Cepeda Duque \\ Facultad de Ciencias Básicas Universidad de Santa Rosa de Cabal, Risaralda, Colombia. acinonyxjubatus96@gmail.com
}

El Mapache Procyon cancrivorus (Cuvier 1798) es un carnívoro mediano de la familia Procyonidae que se distribuye desde el sur de Costa Rica hasta el Norte de Argentina. Habita bosques húmedos, secos, bordes de sabanas y otros hábitats, cerca de ríos y arroyos (De Fatima et al. 1999, Emmons \& Feer 1999, Helgen \& De Wilson 2005, González-Maya et al. 2009). En Colombia, de manera general se distribuye en todo el país, desde la región Caribe y el Chocó biogeográfico, hasta la región amazónica, entre los 0-2350 msnm (Solari et al. 2013, Cuervo-Díaz et al. 1986, Rodríguez-Mahecha et al. 1995, Alberico et al. 2000, Cuartas-Calle \& Muñoz-Arango 2003, Muñoz-Saba \& Alberico 2004, Guzmán-Lenis 2004, Reid \& Helgen 2008, González-Maya et al. 2011, Marín et al. 2012). Aunque es una especie con amplia distribución en el país, son escasos los registros confirmados de esta especie a escala local y regional. Aquí se reporta el primer registro de $P$. cancrivorus para la microcuenca Campoalegrito, municipio de Santa Rosa de Cabal, departamento de Risaralda. Agregando así un nuevo registro local de la especie para Colombia.

La presencia de $P$. cancrivorus fue registrada por medio de rastros (se tomaron las medidas e improntas de las mismas) y de un video obtenido con una cámara trampa (Bushnell Thropy Cam) ubicada en un Agro-ecosistema (café con sombra) dentro del campus de la Universidad de Santa Rosa de Cabal, vereda El Jazmín, municipio Santa Rosa de Cabal, departamento de Risaralda ( $04^{\circ} 54^{\prime} 34.73^{\prime \prime} \mathrm{N} 75^{\circ} 37^{\prime} 29.08^{\prime \prime} \mathrm{W}$ a $1625 \mathrm{msnm}$; figura 1). La zona presenta una precipitación media anual de 1700 a $3100 \mathrm{~mm}$, una humedad relativa del $70 \%$ y una temperatura media mensual de $19.25^{\circ} \mathrm{C}$. Entre la vegetación se pueden encontrar las familias Bignoniaceae, Gesneriaceae, Musaceae, Urticaceae, Poaceae, Zingiberaceae, Meslastomataceae y Rubiaceae. En el lugar se destaca la presencia de árboles frutales del género Psidium y Solanum (Quintela et al. 2014). La especie fue confirmada con base en las descripciones realizadas por varios autores (Vieira 1946; Bisbal 1986, Santos \& Hartz 1999).

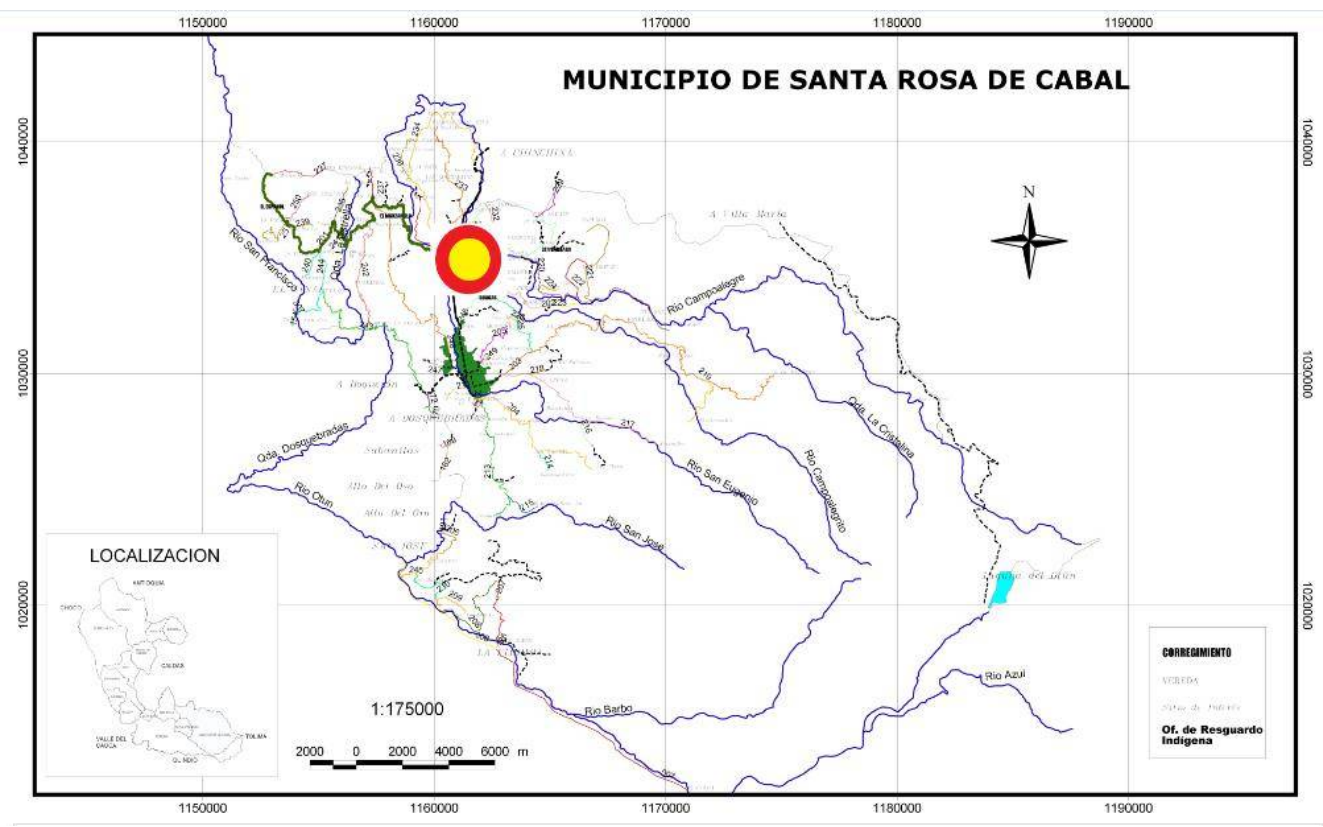

Figura 1. Localización de Procyon cancrivorus en el municipio de Santa Rosa de Cabal, Risaralda, Colombia (Fuente: www.risaralda.gov.co). 
En total se obtuvieron dos registros directos de la especie. El primer registro el 07 de septiembre del 2015 a las $04 \mathrm{~h} 43 \mathrm{hrs}$ y el segundo el 15 de septiembre del 2015 a las 00h43 (Figura 2A, B), en la microcuenca Campoalegrito donde se encuentra el agroecosistema (principalmente monocultivo de café con sombra). Asimismo, se colectaron muestras de cinco huellas de la especie (Tabla 1). Estos registros obtenidos fueron depositados en la colección zoológica de la universidad de Santa Rosa de Cabal. También, en el área se ha registrado la presencia del Zorro Cangrejero Cerdocyon thous (figura 2C, D).
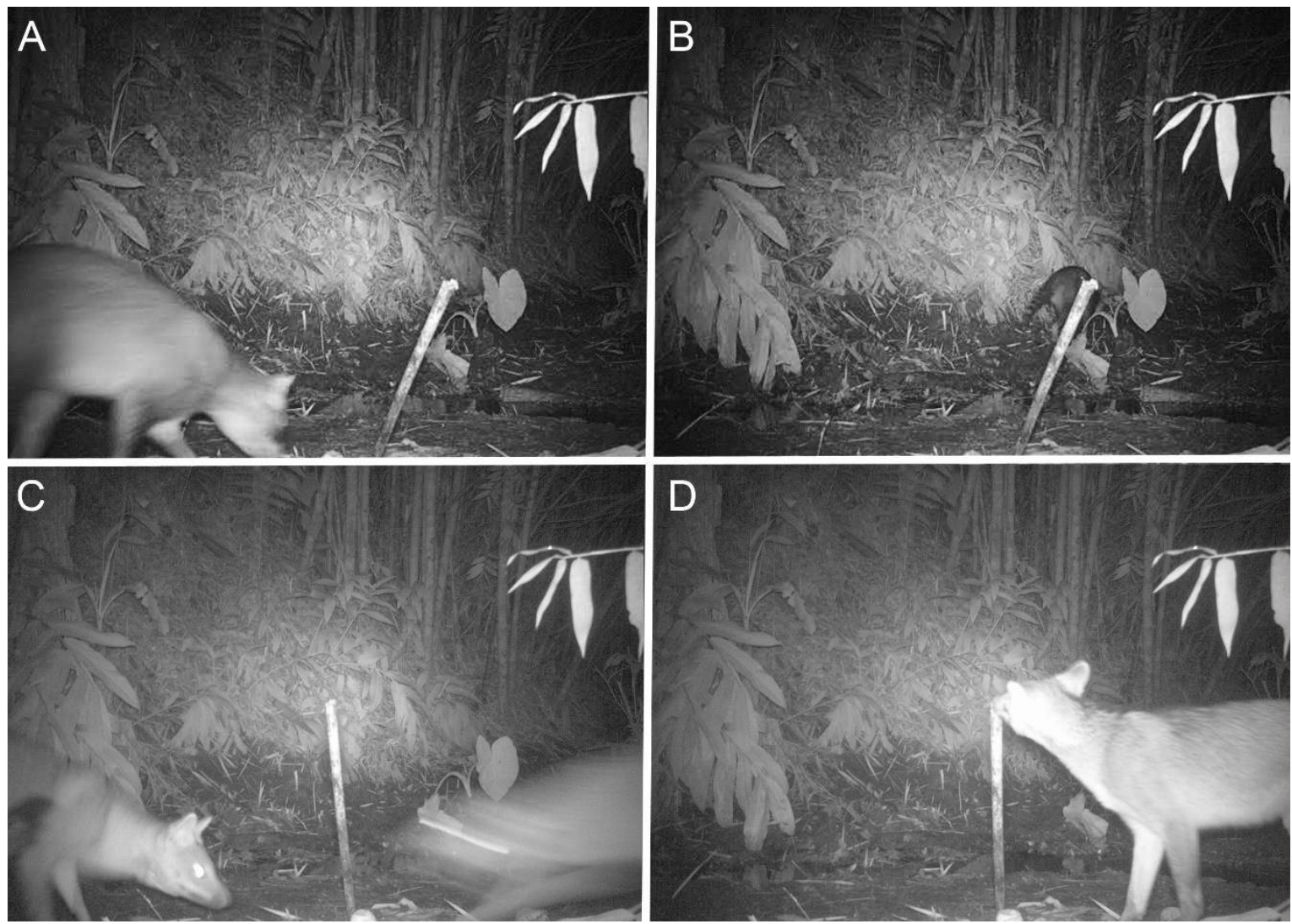

Figura 2. Registro fotográfico de Procyon cancrivorus (A, B) y Cerdocyon thous (C, D) en el campus de la Universidad de Santa Rosa de Cabal, Rirasalda, Colombia

Registros actuales de la especie a nivel local para el país son escasos, sin embargo, se destacan algunos datos para la región Andina en los departamentos de Caldas, Valle del Cauca, Quindío y Antioquia (Marín et al. 2012). Los registros más cercanos de la especie hasta ahora ha sido en los municipios de Victoria $(91.15 \mathrm{Km})$ y Norcasia $(108.60 \mathrm{Km})$ en el departamento Caldas, y en el corregimiento de Santa Elena (114.78Km), Medellín, Antioquía (Marín et al. 2012).

Tabla 1. Medidas de las dimensiones de las huellas de Procyon cancrivorus deposiyadas en la colección zoológica de la Universidad de Santa Rosa de Cabal, Santa Rosa de Cabal, Risaralda.

\begin{tabular}{lccccc}
\hline Número de catálogo & Longitud Huella $(\mathrm{cm})$ & Ancho de Huella $(\mathrm{cm})$ & Longitud de Cojinete $(\mathrm{cm})$ & Ancho de Cojinete $(\mathrm{cm})$ & Garra $(\mathrm{cm})$ \\
\hline CUSM193 & 5.6 & 6.2 & 4.1 & 3.9 & 0.5 \\
\hline CUSM196 & 6.8 & 6.5 & 4.5 & 3.4 & 3.3 \\
\hline CUSM197 & 5.6 & 6.1 & 3.1 & 3.7 & 0.4 \\
\hline CUSM201 & 7.3 & 6.2 & 5.1 & 3.5 & 0.6 \\
\hline CUSM204 & 5.8 & 4.9 & 3.2 & 0.4 \\
\hline
\end{tabular}

Mammalogy Notes | Notas Mastozoológicas

Sociedad Colombiana de Mastozoología

Vol. 3 Num. 1| 2016 
Dadas las características ecológicas de $P$. cancrivorus este tipo de agroecosistemas pueden jugar un papel importante en la conservación de la especie dentro de paisajes fragmentados, ya que estarían suministrando recursos para la especie (Gallina et al. 1996). Por ejemplo, se ha registrado al mapache consumiendo algunos de los frutos de especies del género Psidium y Solanum (Quintinela et al. 2014). Por otra parte, es importante señalar el papel ecológico de esta especie como dispersor de semillas y de control de poblaciones de herbívoros que podrían afectar negativamente estos agroecosistemas (Gatti et al. 2006, Santos \& Hartz 1999, Gatti et al. 2006, Pellanda 2010). Sin embargo, es importante estudiar estas especies de carnívoros en los agroecosistemas, analizando el papel de estas áreas en su conservación y cómo estas especies influyen en la regeneración de los hábitats y en el control de potenciales plagas para estos sistemas productivos. Además, es importante seguir recopilando registros básicos de la especie que permitan completar y afinar su rango de distribución en el país.

Agradecimientos

A John Harold Castaño Salazar por la revisión del material de campo y la confirmación de la especie, Álvaro Botero Botero por sus comentarios en la revisión.

\section{Referencias}

ALBERICO, M., et al. 2000. Mamíferos (Synapsida: Theria) de Colombia. Biota Colombiana, 1(1): 43-75.

BISBAL, F. J. 1986. Food habits of some neotropical carnivores in Venezuela (Mammalia, Carnivora). Mammalia 50: $329-339$.

CUARTAS-CALLE, C. A. \& J. MUÑOZ-ARANGO. 2003. Lista de los mamíferos (Mammalia: Theria) del departamento de Antioquia. Biota Colombiana 4(1): 65-78.

CUERVO-DÍAZ, A., et al. 1986. Lista de los mamíferos de Colombia. Anotaciones sobre su distribución. Caldasia 15 (71-75): 471-502.

DE FÁtiMA, M., et al. 1999. The food habits of Procyon cancrivorus (Carnivora, Procyonidae) in the Lami Biological Reserve, Porto Alegre, Southern Brazil. Mammalia 63: 525-529.

EMMONS, L. \& F. FEER. 1999. Mamíferos de los bosques húmedos de América Tropical. Editorial FAN (Fundación Amigos de la Naturaleza). Santa Cruz, Bolivia.

GATTI, A., et al. 2006. Diet of two sympatric carnivores, Cerdocyon thous and Procyon cancrivorus, in a restinga area of Espírito Santo State, Brazil. Journal of Tropical Ecology 22: 227-230.

GONZÁLEZ-MAYA, J. F., et al. 2009. Activity patterns and community ecology of small carnivores in the Talamanca region, Costa Rica. Small Carnivore Conservation 41: 914.

GONZÁLEZ-MAYA, J. F., et al. 2011. Research priorities for the small carnivores of Colombia. Small Carnivore Conservation, 44: 7-13.

GUZMÁN-LENIS, A. R. 2004. Revisión preliminar de la familia Procyonidae en Colombia. Acta Biológica Colombiana 9(1): 69-76.

HELGEN, K. M. \& DE WILSON. 2005. A systematic and zoogeographic overview of the raccoons of Mexico and Central America. Pp. 219-234 En: Contribuciones Mastozoológicas en homenaje a Bernardo Villa (V Sánchez-Cordero y RA Medellín, eds.). Instituto de Biología e Instituto de Ecología, UNAM.

MARÍN, H. E. R.-C. \& A. F. S.-C. (2012). Revisión craneo-dentaria de Procyon (Carnivora: Procyonidae) en Colombia y Ecuador con notas sobre su taxonomía y distribución. Mastozoologia Neotropical, 19(2), 259-270.

MUÑOZ-SABA, Y. \& M. ALBERICO. 2004. Mamíferos en el Chocó Biogeográfico. Pp. 559-597 En: Colombia. Diversidad Biótica IV. El Chocó Biogeográfico / Costa Pacífica (O Rangel-Ch, ed.). Universidad Nacional de Colombia. Instituto de Ciencias Naturales, Bogotá.

PELlANDA, M. et al. 2010. Dieta do mão-pelada (Procyon cancrivorus, Procyonidae, Carnivora) no Parque Estadual de Itapuã, sul do Brasil. Neotropical Biology and Conservation 5(3): 154-159.

QUINTELA, F. M. et al. 2014. Dieta de Procyon cancrivorus (Carnivora, Procyonidae) en ambientes de restinga e estuarino no Sul do Brasil. Iheringia. Série Zoologia, 104(2): 143-149. http://doi.org/10.1590/1678-476620141042143149

REID, F. \& K. HELGEN.2008. Procyon cancrivorus. En: IUCN Red List of Threatened Species (IUCN 2010, eds.). Version 2010.4.<www.iucnredlist.org>. Acceso 18 abril 2011 RODRÍGUEZ-MAHECHA, J.V. et al. 1995. Mamíferos colombianos: sus nombres comunes e indígenas. Occasional Papers in Coservation Biology No. 3 Conservation International. Santa Fé de Bogotá.

SANTOS, M. \& HARTZ, S. M. 1999.The food habits of Procyon cancrivorus (Carnivora, Procyonidae) in the Lami Biological Reserve, Porto Alegre, Southern Brazil. Mammalia 63: 525-530.

SOLARI, S., et al 2013. Riqueza, endemismo y conservación de los mamíferos de Colombia. Mastozoología Neotropical 20(2): $301-365$

VIEIRA, C. C 1946. Carnívoros do Estado de Sao Paulo. Arquivos de Zoología, 5(3): 135-175.

Mammalogy Notes | Notas Mastozoológicas
Sociedad Colombiana de Mastozoología
Vol. $3 \mathrm{~N}$ um. 1| 2016 